ATLAS Internal Note INDET-NO-105

14 June 1995

\title{
A Life Time Test of Neutron Irradiated Light Emitting Diodes
}

\author{
J. Beringer, K. Borer \\ Laboratory for High Energy Physics, University of Bern, Switzerland \\ C.B. Brooks, A. Fox-Murphy, R.B. Nickerson, A.R. Weidberg \\ Department of Nuclear Physics, Oxford University, UK
}

\begin{abstract}
The results of an accelerated life time test of neutron irradiated ABB Hafo radiation hard LEDs are presented. After a $1 \mathrm{MeV}$ neutron irradiation with up to $1.4 \cdot 10^{14} \mathrm{n} / \mathrm{cm}^{2}$ at the RAL ISIS facility, the LEDs have been operated without seeing any degradation due to ageing for a time which is estimated to correspond to about 57 years of operation in the ATLAS SCT. The irradiation decreased the light output of the LEDs substantially, but during the operation of the LEDs a fast annealing of most of the irradiation damage was observed.
\end{abstract}




\section{Introduction and motivation}

One of the technologies proposed for the optical readout of the ATLAS SemiConductor Tracker (SCT) uses Light Emitting Diodes (LEDs) [1]. Mounted on the detector modules, the LEDs would be subject to an irradiation dose on the order of $10^{5} \mathrm{~Gy}$ and to a $1 \mathrm{MeV}$ neutron equivalent fluence of about $10^{14} \mathrm{n} / \mathrm{cm}^{2}$ in the barrel during 10 years of LHC operation [2]. Recent irradiation tests [3] seem to indicate that sufficiently radiation hard LEDs suitable for fibre optic links are available today.

While it is well known that the light output of LEDs may be decreased by irradiation, little information seems to be available about the influence of radiation on the ageing properties and thus on the life time of LEDs. Without irradiation, ageing processes may lead to degradation and eventually to the failure of a LED. Typical operating life times of commercial unirradiated LEDs under normal operating conditions are on the order of $10^{5}-10^{7}$ hours, and it has been found experimentally that ageing takes place only under forward bias conditions and not when the LED is turned off. The data transmission protocol proposed by the SCT community takes advantage of this fact by switching the LEDs completely off when no data is transmitted.

For the LEDs typically used for fibre optic applications, i.e. high radiance devices operated at high current densities, the dominant degradation process is the inhomogenous development of crystal defects acting as centers for non-radiative recombinations $[4,5,6]$. These defects can be seen under high magnification as dark lines and are therefore often called dark line defects. The growth and propagation of dark line defects starts at initially present material impurities or crystal defects and, by increaseing the non-radiative current, decreases the light output of the LED at a fixed forward current. The rate of growth increases with current density and temperature, but seems to be also enhanced by mechanical stress, e.g. due to diode assembly or dicing-induced strain. The question arises, of course, if radiationinduced displacement damage in LEDs will lead to an increased growth of dark line defects due to the additional crystal defects introduced, and thus to a much shorter life time.

In this note we report on a life time test with a small number of neutron irradiated ABB Hafo LEDs. The goal of this test was more to see if there is any dramatic effect of radiation on the life time of LEDs than to make any statistically significant life time prediction for ATLAS, where only a small fraction of failing LEDs will be acceptable. We are continuing our tests with a larger number of LEDs and with the irradiation including not only neutrons but also charged particles and gammas.

\section{Acceleration of LED ageing}

For most life time tests, the ageing of the components to be studied must be accelerated in order to obtain results within a reasonable time. Under the assumption that the degradation of LEDs takes place only under forward bias, we can achieve an acceleration factor of 12 with respect to LHC simply by operating the LEDs in DC mode. This acceleration factor is based on the estimate that $i$ ) over 10 years, LHC 


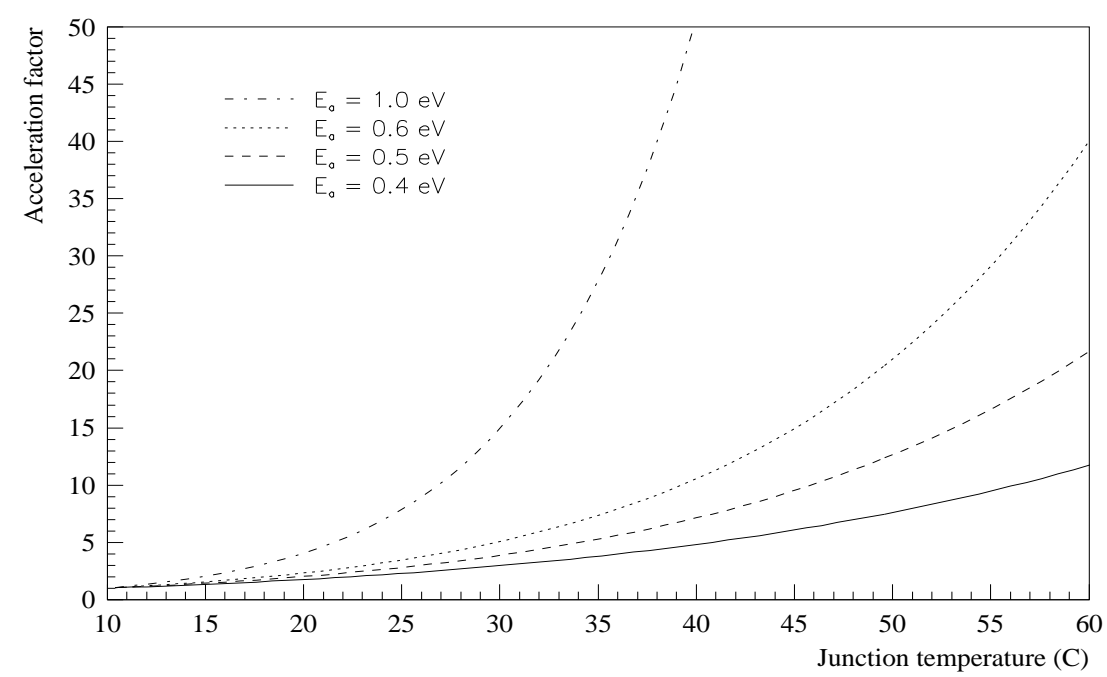

Figure 1: Acceleration factors for different thermal activation energies $E_{a}$, calculated with respect to ageing at $T_{j}=10^{\circ} \mathrm{C}$.

will be running about one third of the time, ii) the average link occupancy should not exceed $50 \%$ to avoid loss of data, iii) the number of 0 and 1 bits is balanced in the data, and $i v$ ) the LEDs are switched off when no data is transmitted.

The ageing of the LEDs can be further accelerated by increasing the operating temperature or the current. The dependence of the mean LED life time $\tau$ on temperature and current is given by $[4,5,6]$

$$
\tau=C I_{f}^{-n} \exp \frac{E_{a}}{k T_{j}}
$$

where $I_{f}$ is the forward current through the LED, $T_{j}$ denotes the junction temperature, and the constants $C, n$ and the thermal activation energy $E_{a}$ depend on the composition and the fabrication process of the LEDs and must be determined experimentally. Values found in the literature for $n$ and $E_{a}$ are typically in the range from 1 to 2 , and from $0.4 \mathrm{eV}$ to $1.0 \mathrm{eV}$, respectively. For some values of $E_{a}$, the acceleration factor that can be gained according to eq. (1) by raising the temperature with respect to ageing at $T_{j}=10^{\circ} \mathrm{C}$ is shown in figure 1 .

\section{Experimental procedure}

Table 1 gives some information about the ABB Hafo radiation hard LEDs used for the life time test. These LEDs, which are not commercially available in the package used for the test, were purchased from GCA Fibreoptics, England, but the manufacturing and the pigtailing with a radiation hard Fujikura 50/125 optical fibre was done by ABB. The ABB Hafo LEDs were chosen by the SDC and RD2 collaborations because of their radiation hardness, their outstanding light yield, and their fast response. 


\begin{tabular}{|l||l|}
\hline LED type & Mini Pigtail LEDs, P/N GCA-90-0174 \\
\hline Light output from fibre & $10 \mu \mathrm{W}-30 \mu \mathrm{W} @ 10 \mathrm{~mA}$ \\
\hline Peak wavelength & $825 \mathrm{~nm}$ \\
\hline Rise and fall time & $4 \mathrm{~ns} @ 10 \mathrm{~mA}$ \\
\hline Pig-tailed package & $\begin{array}{l}\text { Metal cylinder: } L=7.5 \mathrm{~mm}, \oslash=2.5 \mathrm{~mm} \\
\text { Fujikura } 50 / 125 \text { radiation hard fibre }\end{array}$ \\
\hline
\end{tabular}

Table 1: Characteristics of the ABB Hafo radiation hard LEDs.

\begin{tabular}{|l|c|c|}
\hline Sample & LEDs & $\begin{array}{c}1 \mathrm{MeV} \text { neutron dose } \\
\left(10^{14} \mathrm{n} / \mathrm{cm}^{2}\right)\end{array}$ \\
\hline \hline Reference & 8 & No irradiation \\
\hline Low dose & 10 & $0.35 \pm 20 \%$ \\
\hline High dose & 10 & $1.4 \pm 20 \%$ \\
\hline
\end{tabular}

Table 2: Irradiation of the different LED samples.

A total of 28 ABB Hafo LEDs, divided into three samples, have been tested. As shown in table 2, a sample of 8 LEDs was not irradiated and used for reference, and two samples of 10 LEDs each were irradiated with a lower and a higher neutron dose, respectively. Before irradiation, light output, rise and fall time, and the forward voltage for DC operation were measured for all the LEDs at different currents from $1 \mathrm{~mA}$ to $50 \mathrm{~mA}$.

The irradiation took place at RAL, where neutrons with an energy of about $1 \mathrm{MeV}$ are available from the spallation source ISIS [7]. The irradiation dose for each sample is given in table 2 . The uncertainty of $20 \%$ comes from a systematic error. In addition to the $1 \mathrm{MeV}$ neutron fluence, there was a background of low energy neutrons below $10 \mathrm{keV}$ of $2 \cdot 10^{13} \mathrm{n} / \mathrm{cm}^{2}$ and $10^{14} \mathrm{n} / \mathrm{cm}^{2}$ for the lower and the higher dose sample of LEDs, respectively.

After the irradiation, each sample of LEDs was divided evenly into two batches (labelled batch A and batch B) in order to share the work for the long term measurements between Bern and Oxford. The LEDs were mounted in a temperature controlled box and an initial set of measurements was made. The life time test was started by operating the LEDs continuously with a DC current of $10 \mathrm{~mA}$ at an ambient temperature of $8^{\circ} \mathrm{C}\left(T_{j} \approx 10^{\circ} \mathrm{C}\right)$, corresponding to the forward current and the operating temperature expected for the LEDs in the ATLAS SCT. Thus the LED ageing was accelerated by a factor of 12 with respect to ATLAS due to the DC operation (see section 2).

After operating the LEDs for about half an year without seeing any failures, the ageing of the LEDs in batch $\mathrm{A}$ was accelerated further by raising the ambient temperature from $8^{\circ} \mathrm{C}\left(T_{j} \approx 10^{\circ} \mathrm{C}\right)$ to $48^{\circ} \mathrm{C}\left(T_{j} \approx 50^{\circ} \mathrm{C}\right)$, while the ageing of batch $\mathrm{B}$ was continued at an ambient temperature of $8^{\circ} \mathrm{C}$. 


\section{Results}

\subsection{Overview}

The light output of the LEDs after the irradiation versus the DC operating time is shown for the three samples of LEDs in figure 3, 4, and 5. The light output was measured for $10 \mathrm{~mA}$ current pulses and has been normalized with respect to the pre-irradiation light output of each LED. Corrections have been made for the attenuation in optical patch cables and for an average temperature dependence of the light output of $-0.4 \% /{ }^{\circ} \mathrm{C}$. Measurements made after the irradiation before the ageing was started are denoted by small circles and are shown at arbitrary negative values of the time scale.

As expected, the light output of the LEDs in the reference sample (figure 3), which was not irradiated, remained constant throughout the whole test. The RMS spread of the normalized light output of almost $20 \%$ is mainly due to the fact that measurements were made with different optical fibres and connectors, and that for the irradiation all the LEDs were removed from their driver circuit boards. ${ }^{1}$

\subsection{Annealing phase}

After the irradiation, the light output of the LEDs was decreased substantially (table 3). However, during the first few days of DC operation, the light output of most of the LEDs recovered almost entirely (top of figure 4 and 5). The degradation of the light output and the subsequent annealing were correlated with the fall time of light pulses as shown in figure 2, which can be explained with a shorter life time of the minority carriers due to the additional non-radiative recombination centers introduced by the irradiation [8].

\begin{tabular}{|l|c|c|c|c|}
\hline \multirow{2}{*}{ Stage } & \multirow{2}{*}{ Batch } & \multicolumn{3}{|c|}{ Mean normalized light output (\%) } \\
\cline { 2 - 5 } & & Reference & Low dose & High dose \\
\hline \hline Before irradiation & A, B & 100 & 100 & 100 \\
\hline \hline \multirow{2}{*}{ After irradiation } & A & $101 \pm 9$ & $72 \pm 10$ & $36 \pm 5$ \\
\cline { 2 - 5 } & B & $110 \pm 13$ & $73 \pm 6$ & $24 \pm 2$ \\
\hline \multirow{2}{*}{ After 10 days } & A & $98 \pm 10$ & $98 \pm 12$ & $103 \pm 9$ \\
\cline { 2 - 5 } & B & $107 \pm 11$ & $99 \pm 8$ & $67 \pm 2$ \\
\hline \multirow{2}{*}{ At end of test } & A & $101 \pm 10$ & $99 \pm 12$ & $105 \pm 11$ \\
\cline { 2 - 5 } & B & $101 \pm 11$ & $101 \pm 11$ & $79 \pm 6$ \\
\hline \hline After 18 hours at $50 \mathrm{~mA}$ & B & $100 \pm 11$ & $102 \pm 9$ & $87 \pm 8$ \\
\hline
\end{tabular}

Table 3: Mean normalized light output at different stages.

\footnotetext{
${ }^{1}$ Because the LEDs had to be removed from their driver circuit boards for the irradiation, they could not be soldered but were mounted in a mechanical holder, ensuring a good thermal contact. However, it turned out that mechanical pressure on the package of the LEDs could influence the alignement of the optical fibre and thus change the measured light output of the LED. This problem will be avoided in future tests by using a different LED package
} 


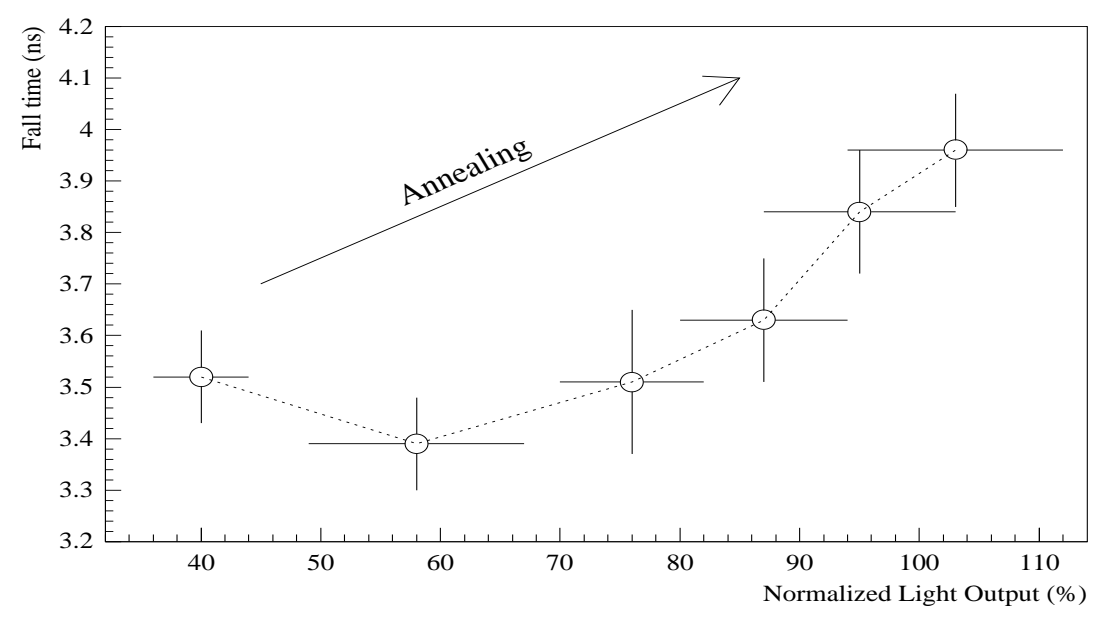

Figure 2: Correlation of the mean normalized light output with the mean fall time for a current step from $10 \mathrm{~mA}$ to $0 \mathrm{~mA}$. Shown are different measurements of batch A made during the annealing phase of the LEDs irradiated with the higher dose. The quadratically added contribution of the current driver, the PIN diode receiver and the oscilloscope to the measured fall time is $3.3 \mathrm{~ns}$.

It is interesting to note the different results obtained for batch $\mathrm{A}$ and for batch $\mathrm{B}$ for the LEDs irradiated with the higher dose of $1.4 \cdot 10^{14} \mathrm{n} / \mathrm{cm}^{2}$. During the first five months after the irradiation, i.e. before the ageing of the LEDs in batch A was accelerated further by raising the temperature to $48^{\circ} \mathrm{C}$, all the LEDs were treated in the same way, except for the initial measurements before the ageing was started: For batch B, the light output was only measured at $10 \mathrm{~mA}$, while for batch A extended measurements were performed, where each LED was operated for about one minute at currents up to $50 \mathrm{~mA}$. As can be seen from the upper plots in figure 4 and 5 , much of the annealing already took place during these initial measurements of batch $A$.

After the first 10 days of continuosly operating the LEDs at $10 \mathrm{~mA}$, a complete annealing of all the irradiated LEDs had been observed in batch $\mathrm{A}$, but in batch $\mathrm{B}$ the LEDs irradiated with the higher dose had recovered only to $67 \%$ of their preirradiation light output (table 3). During the remaining duration of the life time test, the mean normalized light output of these LEDs gradually increased to $79 \%$.

In order to check if a forward current higher than $10 \mathrm{~mA}$ is necessary, at least for a short period of time, to achieve a complete annealing of the irradiation damage ${ }^{2}$, the LEDs in batch B were operated at $50 \mathrm{~mA}$ at the end of the test. During the first few minutes, no significant changes of the light output were observed, but after operating the LEDs for about 18 hours at $50 \mathrm{~mA}$, the mean normalized light output of the LEDs irradiated with the higher dose had increased from $79 \%$ to $87 \%$. The operation of the LEDs at $50 \mathrm{~mA}$ was continued for several days, but the light output did not increase any further.

\footnotetext{
${ }^{2}$ In a recent irradiation test done within the LARG community, no annealing was observed for an ABB Hafo 1A341 LED until the DC current through the LED reached around $100 \mathrm{~mA}$ [3].
} 


\begin{tabular}{|l|c|c||c|c||c|}
\hline Sample & \multirow{2}{*}{ Batch } & \multicolumn{1}{|c||}{ LEDs } & \multicolumn{2}{c||}{ DC operating time (days) at: } & $\begin{array}{c}\text { Corresponding time } \\
\text { in ATLAS (years) }\end{array}$ \\
\cline { 4 - 6 } & & & $8^{\circ} \mathrm{C}, 10 \mathrm{~mA}$ & $48^{\circ} \mathrm{C}, 10 \mathrm{~mA}$ & 57 \\
\hline \hline \multirow{2}{*}{ Reference } & $\mathrm{A}$ & 4 & 162 & 158 & $\mathbf{9}$ \\
\cline { 2 - 5 } & $\mathrm{B}$ & 4 & 272 & $\mathbf{0}$ & 57 \\
\hline \hline \multirow{2}{*}{ Low dose } & $\mathrm{A}$ & 5 & 162 & 158 & $\mathbf{9}$ \\
\cline { 2 - 5 } & $\mathrm{B}$ & 5 & 272 & $\mathbf{0}$ & 57 \\
\hline \hline \multirow{2}{*}{ High dose } & $\mathrm{A}$ & 5 & 162 & 158 & $\mathbf{9}$ \\
\cline { 2 - 5 } & $\mathrm{B}$ & 5 & 272 & $\mathbf{0}$ & \\
\hline
\end{tabular}

Table 4: DC operating time and corresponding estimated operating time in ATLAS.

\subsection{Long-term stability}

After the annealing phase the light output of all LEDs was stable and no degradation due to ageing was observed. Because the parameter $E_{a}$ in eq. (1) is not known for the ABB Hafo LEDs, the additional acceleration factor for the junction temperature $T_{j} \approx 50^{\circ} \mathrm{C}$ during the second phase of the test cannot be determined. If we use for $E_{a}$ the typical values found in the literature from $0.4 \mathrm{eV}$ to $1.0 \mathrm{eV}$, we obtain from eq. (1) a range of acceleration factors from 8 to 160 (see also figure 1). As a conservative estimate we have therefore assumed an additional acceleration factor of 10 for the ageing at $T_{j} \approx 50^{\circ} \mathrm{C}$.

For each sample of LEDs, the DC operating time after irradiation is summarized in table 4 . The last column gives an estimate for the corresponding operating time in ATLAS. The estimate takes into account an acceleration factor of 12 due to DC operation and, for the LEDs operated at $T_{j} \approx 50^{\circ} \mathrm{C}$, the additional factor of 10 as explained above. As can be seen, the LEDs irradiated with the higher dose of $1.4 \cdot 10^{14} \mathrm{n} / \mathrm{cm}^{2}$ have been operated for a time corresponding to about 57 years (batch A) and 9 years (batch B) of operation at LHC without seeing any degradation.

\section{Conclusions}

After a $1 \mathrm{MeV}$ neutron irradiation with up to $1.4 \cdot 10^{14} \mathrm{n} / \mathrm{cm}^{2}$, we have operated ABB Hafo LEDs in an accelerated life time test for up to 320 days without seeing any degradation due to ageing. The duration of the test is estimated to correspond to about 57 years of operation in the ATLAS SCT. Therefore, in spite of the limited statistics, the possibility that neutron irradiation drastically reduces the life time of LEDs due to a much increased growth of dark line defects may be excluded for the radiation levels at $\mathrm{LHC}$.

As expected, the light output of the LEDs was decreased substantially by the irradiation. During the operation of the LEDs, a complete annealing of the irradiation damage was observed in batch $\mathrm{A}$ within the first 10 days. In batch $\mathrm{B}$ however, until the end of the life time test, the LED sample irradiated with the higher dose had only recovered to $79 \%$ of its pre-irradiation light output. By operating the LEDs at $50 \mathrm{~mA}$ for about 18 hours, the mean normalized light output of this LED sample could be increased to $87 \%$, but a complete annealing was not achieved. 
The results obtained so far are very encouraging. However, in order to make a reliable prediction for ATLAS, more statistics are definitely needed, the radiation damage caused by charged particles and gammas should be examined as well, and the conditions required for the annealing of the irradiation damage need to be clarified. A continuation of our tests in this direction is in progress.

\section{References}

[1] SCT group, ATLAS SCT Technical Proposal Backup Document, ATLAS Internal Note, INDET-NO-085 (1995)

[2] ATLAS Collaboration, Technical Proposal, CERN/LHCC/94-43 (1994)

[3] B. Dinkespiler et al., Neutron Irradiation of Optical Link Components, ATLAS Internal Note, LARG-NO-002 (1994)

[4] M.H. Pilkuhn, Light Emitting Diodes, in T.S. Moss, Handbook on Semiconductors, Volume 4, North-Holland (1981)

[5] Honeywell, Long-Term Power Output Reliability of Cap Rock (SEC589) LED Chip, Optoelectronics Fiber Optic Products, Catalog E27 (1993)

[6] Hewlett Packard, Reliability Considerations in Designing Fiber Optic Transmitters, Optocoupler and Fiber Optics Applications Handbook (1986)

[7] M. Edwards and D.R. Perry, The Radiation Hardness Test Facility, RAL Report, RAL-90-065 (1990)

[8] C.E. Barnes, Radiation Effects on Light Sources and Detectors, SPIE Vol. 541, Radiation Effects in Optical Materials (1985) 

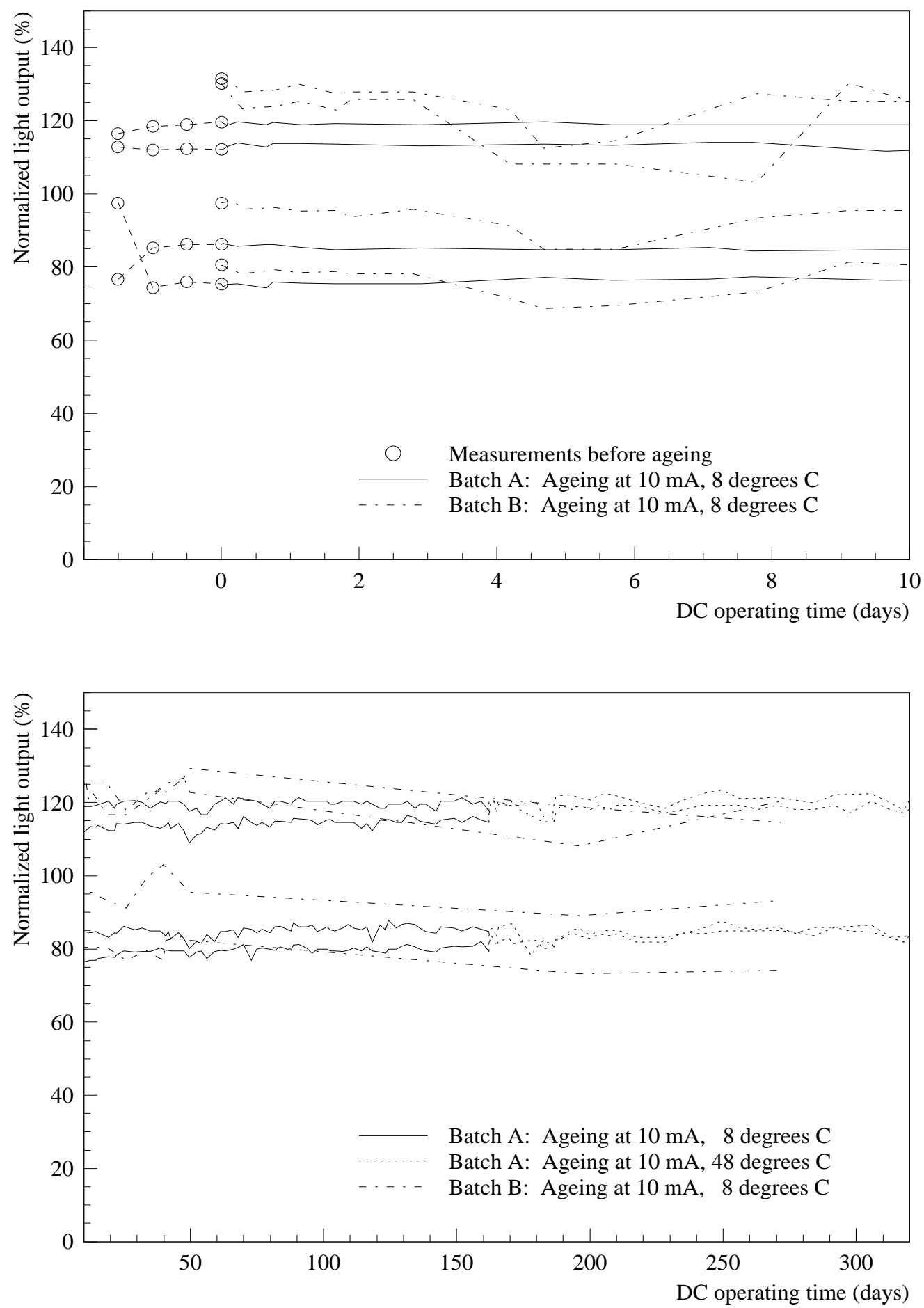

Figure 3: Normalized light output of the reference LEDs which were not irradiated. Measurements made before the ageing was started (denoted by small circles at arbitrary negative values of the time scale), and the light output measured during the first 10 days of DC operation are shown on the top. The light output after the first 10 days is displayed on the bottom. The drop in the light output of the LEDs in batch $\mathrm{B}$ between days 5 and 8 was caused by a faulty optical connector. 

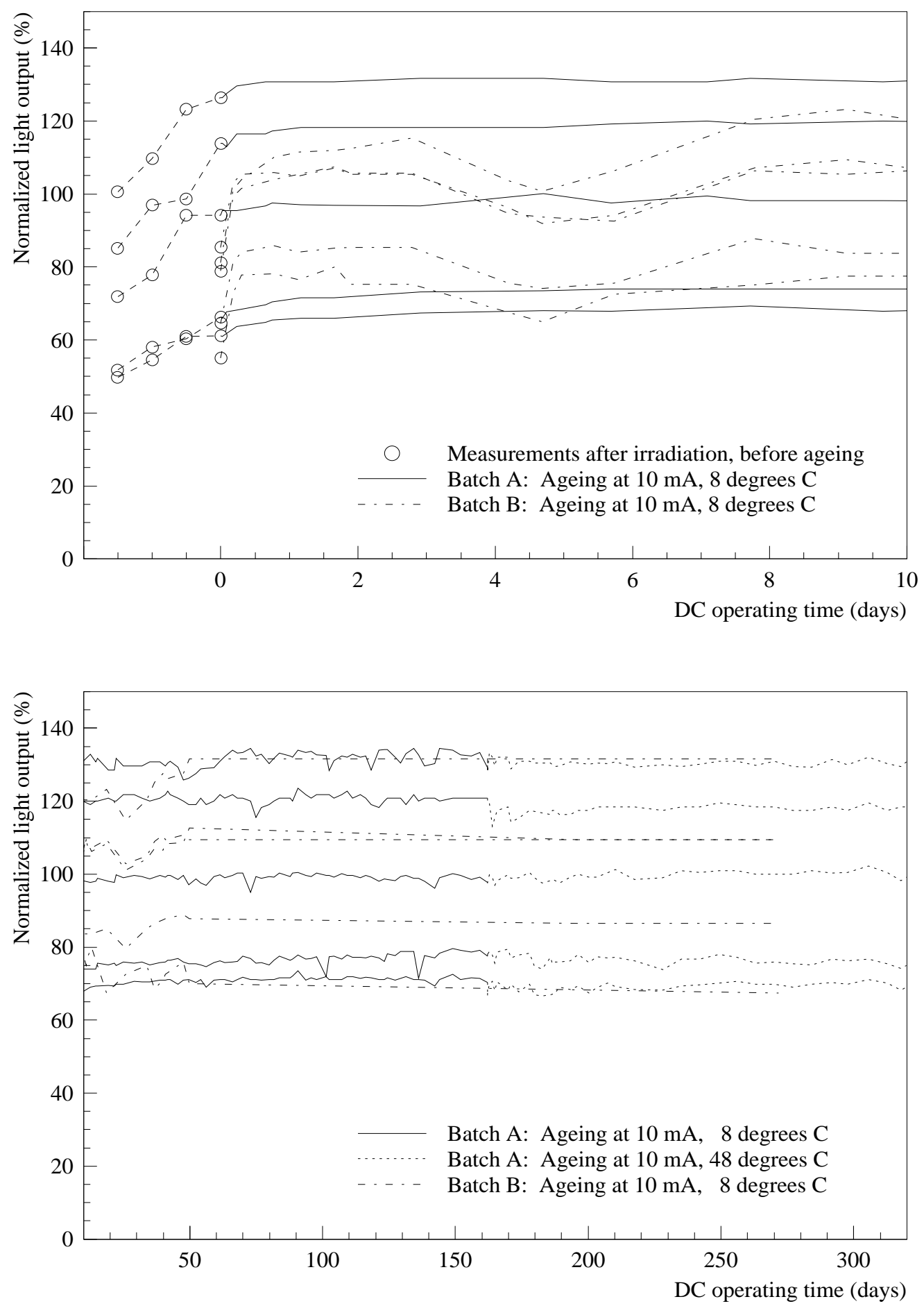

Figure 4: Normalized light output of the LEDs irradiated with the lower $1 \mathrm{MeV}$ neutron dose of $3.5 \cdot 10^{13} \mathrm{n} / \mathrm{cm}^{2}$. Measurements made after irradiation before the ageing was started (denoted by small circles at arbitrary negative values of the time scale), and the light output measured during the first 10 days of DC operation (annealing phase) are shown on the top. The light output after the annealing phase is displayed on the bottom. The drop in the light output of the LEDs in batch B between days 5 and 8 was caused by a faulty optical connector. 

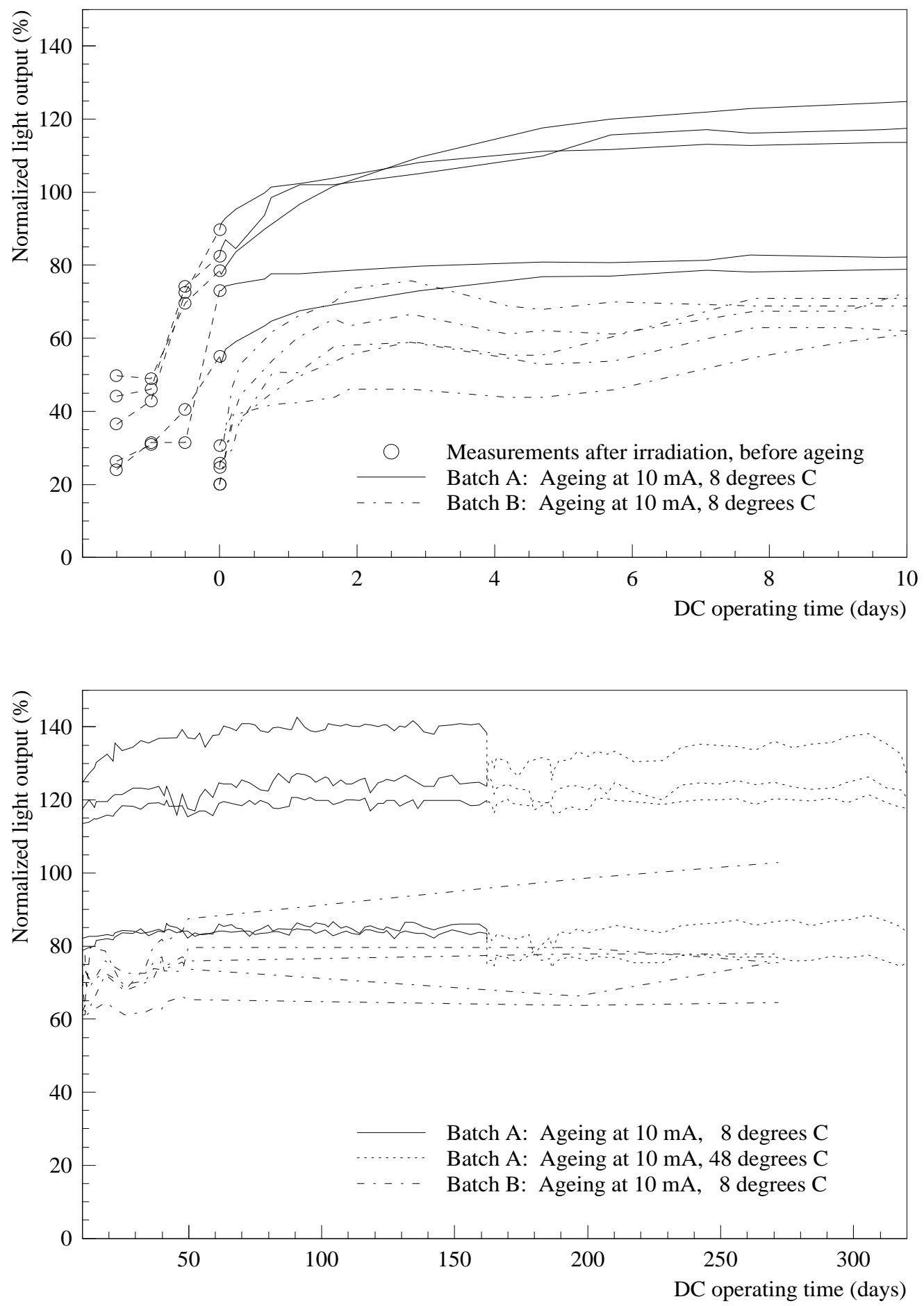

Figure 5: Normalized light output of the LEDs irradiated with the higher $1 \mathrm{MeV}$ neutron dose of $1.4 \cdot 10^{14} \mathrm{n} / \mathrm{cm}^{2}$. Measurements made after irradiation before the ageing was started (denoted by small circles at arbitrary negative values of the time scale), and the light output measured during the first 10 days of DC operation (annealing phase) are shown on the top. The light output after the annealing phase is displayed on the bottom. The drop in the light output of the LEDs in batch B between days 5 and 8 was caused by a faulty optical connector. 